\title{
RELAXATION DUE TO INCOHERENT TUNNELLING IN DIELECTRIC GLASSES
}

\author{
Peter Neu and Alois Würger \\ Institut für \\ Theoretische Physik \\ Universität Heidelberg \\ Philosophenweg 19 \\ 69120 Heidelberg, Germany
}

\begin{abstract}
A new relaxation mechanism is shown to arise from overdamped two-level systems above a critical temperature $T^{*} \approx 5 \mathrm{~K}$, thus yielding an explanation for experimental observations in dielectric glasses in the temperature range between $T^{*}$ and the relaxation peak at $50 \mathrm{~K}$. Using the distribution function of the tunnelling model for the parameters of the two-level systems, both the linear decrease of the sound velocity and the linear increase of the absorption up to the relaxation maximum, are quantitatively accounted for by our theory.
\end{abstract}

PACS. 61.40- amorphous and polymeric materials

PACS. 63.50 - disordered solids, vibrational states

PACS. 77.22G - relaxation phenomena, dielectrics 
Low temperature properties of glasses below 1 Kelvin [四] are satisfactorily explained by the assumption of localised tunnelling states (TS) with a wide distribution of energies and relaxation times; these TS are commonly described by a mapping on two-level systems (TLS) [2]. Usually only the direct (one-phonon) relaxation mechanism of TS with phonons is considered [3]. For this reason there is poor agreement with experiment at temperatures above a few Kelvin [4,5.6]; especially the linear increase of the absorption up to the relaxation peak at about $50 \mathrm{~K}$ and the linear decrease with temperature of the sound velocity seem to be a universal characteristic of amorphous substances [4, 5, 6]. There are attempts to explain that linear temperature variation by thermally activated processes [7],8], or by elastic anharmonicity of the lattice [6], or by a modification of the standard distribution function for the tunnelling parameters [5].

In this letter we provide an explanation for the temperature variation above a few Kelvin which relies on the two-level description and the standard distribution function, thus avoiding introduction of new parameters. We find in this temperature regime incoherent tunnelling rather than coherent oscillations; as a result, all TLS, even the symmetric ones, contribute to relaxation and therefore yield a more pronounced temperature dependence of sound and microwave propagation. In this paper we extend our previous treatment of symmetric TS [9] to the biased case.

The model is described by the spin-boson Hamiltonian [10]

$$
H=-\frac{1}{2} \hbar \Delta_{0} \sigma_{x}+\frac{1}{2} \hbar \Delta \sigma_{z}+\gamma e \sigma_{z}+H_{B}
$$

where $\Delta_{0}$ denotes the tunnelling amplitude, $\Delta$ the bias, $\gamma$ the deformation potential, and

$$
e=i \sum_{k} k \sqrt{\frac{\hbar}{2 m_{k} \omega_{k}}}\left(b_{k}-b_{k}^{\dagger}\right)
$$

the distortion of the lattice. We consider the coupling of the TS to three-dimensional acoustic phonons $\left(\omega_{k}=k v\right)$ described by $H_{B}=\sum_{k} \hbar \omega_{k} b_{k}^{\dagger} b_{k}$ and bosonic operators fulfilling $\left[b_{k}, b_{k^{\prime}}^{\dagger}\right]=\delta_{k, k^{\prime}}$. The model is specified by the spectral density which in Debye approximation is given by

$$
J(\omega)=\frac{4}{\hbar} \sum_{k} \frac{\gamma^{2} \omega_{k}}{2 m_{k} v^{2}} \delta\left(\omega-\omega_{k}\right)=\tilde{\gamma}^{2} \omega^{3} \exp \left(-\omega / \omega_{D}\right)
$$

where $\tilde{\gamma}^{2}=\gamma^{2} /\left(\pi^{2} \varrho v^{5} \hbar\right)$, and $\omega_{D}$ is the Debye frequency, $\varrho$ the mass density and $v$ the sound velocity. In the tunnelling model the parameters $\Delta_{0}$ and $\Delta$ are assumed to be distributed according to $P\left(\Delta_{0}, \Delta\right)=\bar{P} / \Delta_{0}$, which is equivalent to

$$
P(\epsilon, r) \mathrm{d} \epsilon \mathrm{d} r=\frac{\bar{P}}{2 r \sqrt{1-r}} \mathrm{~d} \epsilon \mathrm{d} r
$$

with a constant $\bar{P}$ and new parameters $r=\Delta_{0}^{2} / \epsilon^{2}$ and $\epsilon=\sqrt{\Delta_{0}^{2}+\Delta^{2}}$, where $r_{\min } \leq r \leq 1$.

All dynamical information is contained in the symmetrized two-time correlation function $C_{z z}(t)$, which is calculated in the framework of the Mori-Zwanzig projection formalism [11] 
using a mode-coupling approximation [9, 12]. With the projector $\left.\mathcal{P}=\sum_{\alpha} \mid \sigma_{\alpha}\right)\left(\sigma_{\alpha} \mid=\mathcal{I}-\mathcal{Q}\right.$ and the scalar product $(A \mid B)=\operatorname{tr}\left[\rho_{e q}(1 / 2)(A B+B A)\right]$, the equilibrium density matrix $\rho_{e q}=$ $\exp (-\beta H) / \operatorname{tr}(\exp (-\beta H))$, the resolvent matrix $C_{\alpha \beta}(z)=\left(\sigma_{\alpha}\left|[\mathcal{L}-z]^{-1}\right| \sigma_{\beta}\right)(\alpha=x, y, z)$ of the Liouvillian $\mathcal{L} *=[H, *] / \hbar$ can be written as

$$
\left[z \delta_{\alpha \beta}-\Omega_{\alpha \beta}+M_{\alpha \beta}(z)\right] C_{\beta \gamma}(z)=-\delta_{\alpha \gamma}
$$

Here $\Omega_{\alpha \beta}=\left(\sigma_{\alpha}|\mathcal{L}| \sigma_{\beta}\right)$ is the frequency matrix and

$$
M_{\alpha \beta}(z)=\left(\mathcal{Q} \mathcal{L} \sigma_{\alpha}\left|[\mathcal{Q} \mathcal{L} \mathcal{Q}-z]^{-1}\right| \mathcal{Q} \mathcal{L} \sigma_{\beta}\right)=\left(\begin{array}{ccc}
\Sigma_{y y}(z) & -\Sigma_{y x}(z) & 0 \\
-\Sigma_{x y}(z) & \Sigma_{x x}(z) & 0 \\
0 & 0 & 0
\end{array}\right)
$$

is the damping matrix with the spin-phonon resolvent $\Sigma_{\alpha \beta}(z)=\left(e \sigma_{\alpha}\left|[\mathcal{Q L \mathcal { Q }}-z]^{-1}\right| e \sigma_{\beta}\right)$. In mode-coupling approximation the memory functions are decoupled according to [9.12

$$
\Sigma_{\alpha}^{\prime \prime}(\omega)=C_{\alpha}^{\prime \prime}(\omega) * \tilde{J}(\omega)
$$

for $\alpha=x, y, z, a, s$, where we have defined the bath spectral function $\tilde{J}(\omega)=J(\omega)$ $\operatorname{coth}(\beta \hbar \omega / 2)$, the weighted convolution integral

$$
g(\omega) * h(\omega)=\int \frac{\mathrm{d} \omega^{\prime} \cosh (\beta \hbar \omega / 2)}{2 \cosh \left(\beta \hbar \omega^{\prime} / 2\right) \cosh \left(\beta \hbar\left(\omega-\omega^{\prime}\right) / 2\right)} g\left(\omega-\omega^{\prime}\right) h\left(\omega^{\prime}\right)
$$

and the resolvent functions $C_{\alpha}(z)=C_{\alpha \alpha}$ for $\alpha=x, y, z$, and $C_{a}(z)=-i\left(C_{x y}(z)-C_{y x}(z)\right)$ and $C_{s}(z)=C_{x y}(z)+C_{y x}(z)$. Here the imaginary parts of the resolvent functions, i. e. the spectral functions, are indicated by a double prime; the real parts are obtained from these via a Kramers-Kronig relation. By noting $C_{y}^{\prime \prime}(\omega)=\left(\omega / \Delta_{0}\right)^{2} C_{z}^{\prime \prime}(\omega)$ eq. (5. - 7) get closed and can be solved numerically by iteration. They show a transition from coherent tunnelling, where $C_{z}^{\prime \prime}(\omega)$ has resonances at $\omega \approx \pm \epsilon$ and $\omega=0$, to incoherent tunnelling motion, where the three resonances have merged in one single resonance at $\omega=0$ whose width narrows with further rising temperature. In both asymptotic regimes an analytic solution of eq. (5 - 7) is possible.

(i) In the coherent or weak-coupling regime first Born-approximation is reliable; after replacing in (7) $C_{\alpha}^{\prime \prime}(\omega)$ by the free spin-spectral function, and also discarding the spin-phonon interaction in $\rho_{e q}$, one easily derives the well-known results (cf. [13])

$$
\begin{aligned}
C_{z}^{\prime \prime}(\omega) & =\pi\left\langle\sigma_{z}\right\rangle^{2} \delta(\omega), \\
& +\frac{1-r}{\cosh ^{2}(\beta \hbar \epsilon / 2)} \frac{\Gamma_{1}}{\omega^{2}+\Gamma_{1}^{2}}+\frac{r}{2}\left[\frac{\Gamma_{2}}{(\omega-\epsilon)^{2}+\Gamma_{2}^{2}}+\frac{\Gamma_{2}}{(\omega+\epsilon)^{2}+\Gamma_{2}^{2}}\right]
\end{aligned}
$$

with the usual one-phonon rate

$$
\Gamma_{1} \equiv 2 \Gamma_{2}=r \frac{\pi}{2} \tilde{\gamma}^{2} \epsilon^{3} \operatorname{coth}(\beta \hbar \epsilon / 2)
$$

(ii) In the incoherent or strong-coupling regime the full dynamics in $C_{z}^{\prime \prime}(\omega)$ and $\rho_{e q}$ is kept and the eq. (5 - 7) are treated self-consistently. Off-diagonal correlations like $\Sigma_{x y}(z)$ 
and spin-polarisations $\left\langle\sigma_{x}\right\rangle,\left\langle\sigma_{z}\right\rangle$ are now negligible. The relevant singularities of $C_{z}(z)$ are at $z_{ \pm}= \pm \Omega-i \Gamma_{2}, z_{0}=-i \Gamma_{1}$ where $\Omega \approx \Delta, \Gamma_{2} \approx \tilde{\Gamma}, \Gamma_{1} \approx \Delta_{0}^{2} / \tilde{\Gamma}$ asymptotically. Here we have identified $\Sigma_{x}(z)$ with $\Sigma_{y}(z)$ and have defined $\tilde{\Gamma}$ by $i \tilde{\Gamma}=\Sigma_{x}(z=0)$. For $\tilde{\Gamma} \gg \epsilon$ the oscillating poles have zero residue, so that the asymptotic form of the relevant spectral function reads

$$
C_{z}^{\prime \prime}(\omega)=\frac{\Gamma_{1}}{\omega^{2}+\Gamma_{1}^{2}}
$$

Inserting this in the mode-coupling integral (17) yields

$$
\tilde{\Gamma}=\frac{\pi^{2}}{2} \tilde{\gamma}\left(\frac{k_{B} T}{\hbar}\right)^{2}
$$

For low-frequency acoustic experiments on glasses, only the relaxational pole at $\omega=0$ is relevant. For this pole the following formulae reasonably interpolate between the behaviour in the coherent $(9-10)$ and the incoherent regime (11 - 12)

$$
C_{\mathrm{rel}}^{\prime \prime}(\omega)=\frac{\tilde{\Gamma}^{2}+\Delta^{2} / \cosh ^{2}(\beta \hbar \epsilon / 2)}{\tilde{\Gamma}^{2}+\epsilon^{2}} \frac{\Gamma_{1}}{\omega^{2}+\Gamma_{1}^{2}}
$$

with the relaxation rate

$$
\Gamma_{1}=\frac{r \epsilon^{2} \tilde{\Gamma}}{\tilde{\Gamma}^{2}+\epsilon^{2}}=: \frac{r}{\tau_{\min }}
$$

$\tau_{\text {min }}$ being independent of $r$, and

$$
\tilde{\Gamma}= \begin{cases}\frac{\pi}{2} \tilde{\gamma}^{2} \epsilon^{3} \operatorname{coth}(\beta \hbar \epsilon / 2)=: \tilde{\Gamma}_{1 \mathrm{ph}} & T<T^{*} \\ \frac{\pi^{2}}{2} \tilde{\gamma}\left(\frac{k_{B} T}{\hbar}\right)^{2}=: \tilde{\Gamma}_{\mathrm{MC}} & T \geq T^{*}\end{cases}
$$

Here $T^{*}$ is the temperature were all thermal TLS $\left(\hbar \epsilon \leq k_{B} T\right)$ are overdamped. The condition $\tilde{\Gamma}_{\mathrm{MC}}\left(T^{*}\right) \equiv k_{B} T^{*} / \hbar$ yields the transition temperature

$$
T^{*}=\frac{2 \hbar}{\pi^{2} k_{B}} \frac{1}{\tilde{\gamma}}
$$

Internal friction and variation of sound velocity are given by [1]

$$
\begin{aligned}
Q^{-1} & =\frac{\gamma^{2}}{\varrho v^{2}} \overline{\chi^{\prime \prime}(\omega)} \\
\frac{\delta v}{v} & =-\frac{1}{2} \frac{\gamma^{2}}{\varrho v^{2}} \overline{\chi^{\prime}(\omega)}
\end{aligned}
$$

the absorbative part $\chi^{\prime \prime}(\omega)$ of the dynamical susceptibility is related to the fluctuating part of the spectral function by the fluctuation-dissipation theorem. The bar denotes 
the average over tunnelling systems with respect to (4). By cutting the $\epsilon$-integration at $\epsilon=\max \left(k_{B} T / \hbar, \tilde{\Gamma}\right)$ one finds for the internal friction

$$
Q^{-1}= \begin{cases}\frac{8 \pi C \tilde{\gamma}^{2} k_{B}^{3} T^{3}}{9 \omega \hbar^{3}} & T<T_{\text {res }}^{*} \\ \frac{\pi}{2} C\left(1-\frac{\hbar \tilde{\Gamma}_{\mathrm{MC}}}{2 k_{B} T} \arctan \left(\frac{2 k_{B} T}{\hbar \tilde{\Gamma}_{\mathrm{MC}}}\right)\right) & \\ +\frac{\pi}{2} C \frac{\hbar \tilde{\Gamma}_{\mathrm{MC}}}{2 k_{B} T}\left(\frac{\pi}{2}-\arctan \left(\frac{\tilde{\Gamma}_{\mathrm{MC}} r_{\min }}{\omega}\right)\right), & T>T_{\mathrm{res}}^{*}\end{cases}
$$

and - by adding the contribution of the resonant part $\delta v /\left.v\right|_{\text {res }}=C \ln \left(T / T_{0}\right)$ - for the change of the sound velocity

$$
\frac{\delta v}{v}= \begin{cases}C \ln \frac{T}{T_{0}} & T<T_{\mathrm{res}}^{*} \\ -\frac{1}{2} C \ln \frac{T}{T_{0}} & T_{\mathrm{res}}^{*}<T<T^{*} \\ -\left(\frac{1}{4}+\frac{\pi}{16}\right) C \frac{T}{T^{*}} \ln \left(\frac{T^{2}}{T^{*} \hbar \omega / k_{B}}\right) . & T>T^{*}\end{cases}
$$

with $C=\bar{P} \gamma^{2} / \varrho v^{2} . T_{\text {res }}^{*}$ separates the regimes where the resonant $\left(T<T_{\text {res }}^{*}\right)$ and the relaxational process $\left(T>T_{\text {res }}^{*}\right)$ prevails. Below $T^{*}$ one finds the well-known logarithmic temperature dependence of the sound velocity and the constant internal friction. At $T=T^{*}$ the temperature dependence changes to a linear increase in the absorption and a linear decrease in the sound velocity.

From a recent experiment on Suprasil W [14 one finds a transition from the plateau to the linear increase at about $T^{*} \approx 6 \mathrm{~K}$ which corresponds according to (16) to a deformation potential of about $\gamma \approx 2 \mathrm{eV}$. Here we have used $C=2.8 \times 10^{-4}[1], \varrho=2.2 \mathrm{~g} / \mathrm{cm}^{3}$, $v_{\ell}=5.8 \times 10^{5} \mathrm{~cm} / \mathrm{sec}, v_{t}=3.75 \times 10^{5} \mathrm{~cm} / \mathrm{sec}$ [3] and $\gamma_{\ell}^{2} \approx 2 \gamma_{t}^{2}$ with $\gamma^{2} / v^{5}=\gamma_{\ell}^{2} / v_{\ell}^{5}+2 \gamma_{t}^{2} / v^{5}$. With these values we calculate the slope with respect to temperature; for a comparison with experiment see Table 1. Both the prefactor and the logarithmic variation of the sound velocity with frequency show full agreement.

In Fig. 1-2 we have plotted our theoretical results together with the experimental data for Suprasil W [14. At temperatures between $100 \mathrm{mK}$ and $15 \mathrm{~K}$ there is full agreement between experiment and theory; for both absorption and sound velocity the measured data could be reproduced with the same numerical values for $\tilde{\gamma}$ and $C$. A similarly good agreement has been found at other frequencies and in recent experiments on $\mathrm{GeO}_{2}$ [15].

Finally, we comment on the absorption peak at about $30 \mathrm{~K}$. According to (19) we find in our theory $T_{\max } \propto \sqrt{\omega}$. However, experimentally the frequency dependence of the relaxation maximum is found to be much weaker [14]. This would indicate the onset of thermally activated processes to occur at some temperature below $T_{\max }$ which yields a logarithmical variation with frequency, rather than the square root dependence. For that case the quoted value of $r_{\text {min }}$ in Fig. 1 has no physical relevance. We stress that this does not affect the temperature variation below $T_{\max }$.

In summary, we have shown that the experimental data in the absorption and the sound velocity up to the relaxation peak can be explained in the framework of the tunnelling model. The novel features arise from the incoherent dynamics of the tunnelling motion at temperatures above $T^{*}$. In particular, this provides a new relaxation mechanism which accounts well for the experimental findings in glasses above $5 \mathrm{~K}$. 


\section{ACKNOWLEDGEMENT}

We are grateful to Johannes Classen, Christian Enss and Sonja Rau for helpful discussions and for kindly communicating experimental data prior to publication. 


\section{REFERENCES}

[1] Hunklinger, S., Arnold, W.: In: Physical acoustics, vol. 12, Thurston R. N., Mason W. P. (eds). New York: Academic Press (1976); Hunklinger, S., Raychaudhuri, A. K.: In: Progress in low temperature physics, vol. IX, Brewer D. F. (ed.). Amsterdam: Elsevier (1986)

[2] Anderson, P. W., Halperin, B. I., Varma, C.: Philos. Mag. 25, 1 (1972); Phillips, W. A.: J. Low. Temp. Phys. 7, 351 (1972)

[3] Jäckle, J.: Z. Phys. 257, 212 (1972)

[4] Krause, J. T.: J. Appl. Phys. 42, 3035 (1971); Bellessa, G., Lemercier, C., Caldemaison, D.: Phys. Lett. 62A, 127 (1977); Bellesa, G.: Phys. Rev. Lett. 40, 1456 (1978)

[5] Anthony, P. J., Anderson, A. C.: Phys. Rev. B 20, 763 (1979)

[6] Doussineau, P., Frenois, C., Leisure, R. G., Levelut, A., Prieur, J.-Y.: J. Physique 41, 1193 (1980)

[7] Tielburger, D., Merz, R., Ehrenfels, R., Hunklinger, S.: Phys. Rev. B 45, 2750 (1992)

[8] Buchenau, U., Galperin, Yu. M., Gurevich, V. L., Parshin, D. A., Ramos, M. A., Schober, H.R.: Phys. Rev B 46, 2798 (1992)

[9] Neu, P., Würger, A.: appears in Z. Phys. B

[10] Leggett, A. J., Chakravarty, S., Dorsey, A. T., Fisher, M. P. A., Garg, A., Zwerger, W.: Rev. Mod. Phys. 59, 1 (1987); Weiss, U.: Quantum Dissipative Dynamics, Series in Modern Condensed Matter Physics, Vol. 2, World Scientific, Singapore (1993)

[11] Mori, H.: Progr. Theor. Phys. 33, 127 (1965); Zwanzig, R.: J. Chem. Phys. 33, 1338 (1960)

[12] Beck, R., Götze, W., Prelovsek, P.: Phys. Rev A 20, 1140 (1979); Zwerger, W.: Z. Phys. B 53, 53 (1983); ibid 54, 87 (1983)

[13] Pirc, R., Gosar, P.: Phys. kondens. Materie 9, 377 (1969)

[14] Classen, J., Enss, C., Bechinger, C., Weiss, G., Hunklinger, S.: appears in Annalen der Physik (1994)

[15] Rau, S.: Private communication (1994) 


\section{FIGURES}
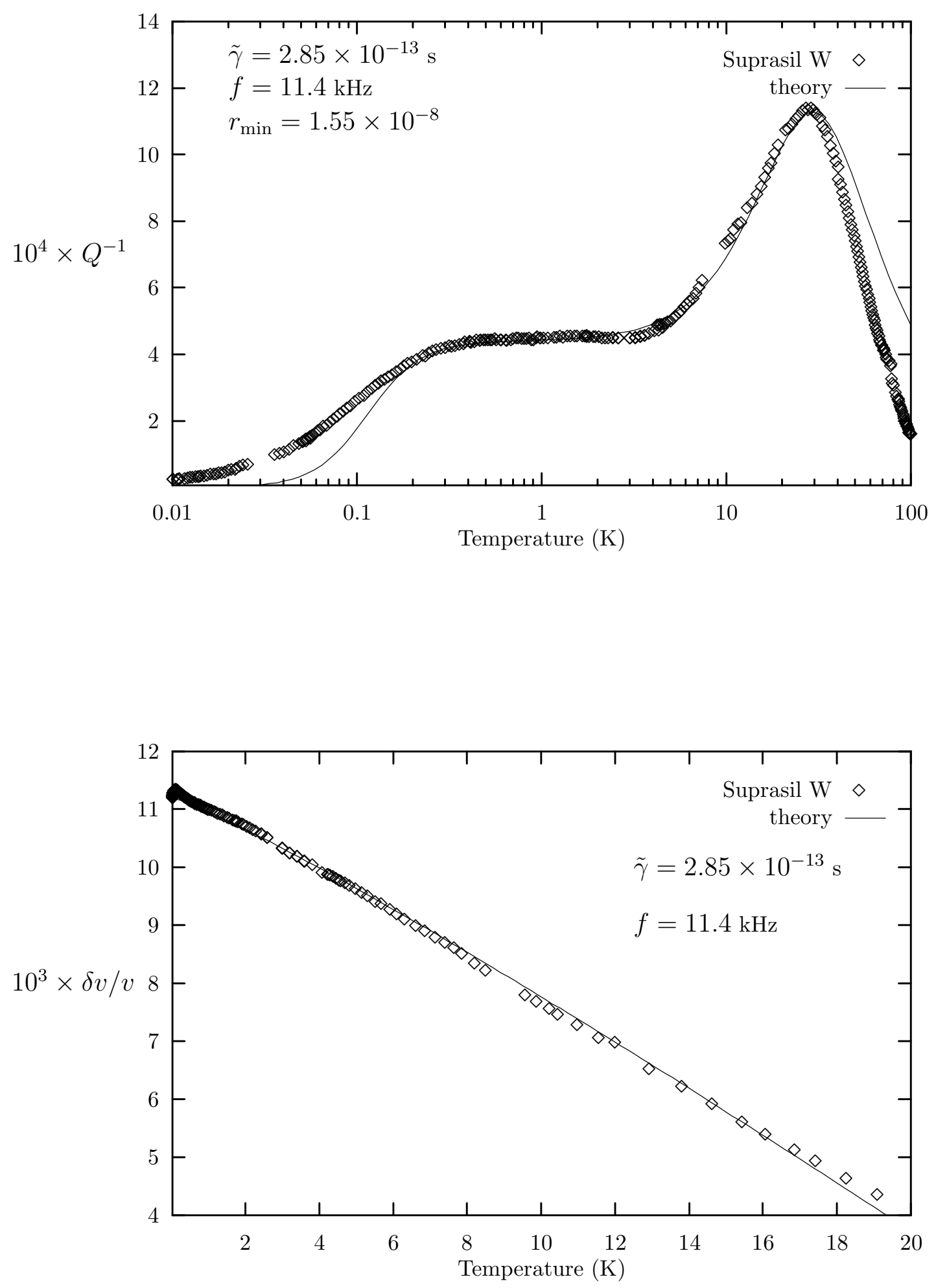


\section{CAPTIONS}

Fig. 1: Internal friction for Suprasil $\mathrm{W}$ at $11.4 \mathrm{kHz}$. The data $(\diamond)$ are from Classen et al. 114. In our theory (-) we have used $C=2.8 \times 10^{-4}$ and $\gamma_{\ell}=2.2 \mathrm{eV}$

Fig. 2: Relative variation of the sound velocity for Suprasil W at $11.4 \mathrm{kHz}$. The data $(\diamond)$ are from Classen et al. 114. In our theory $(-)$ we have used the same numerical value for $C$ and $\gamma_{\ell}$ as in Fig. 1 


\section{TABLES}

Table 1: Comparison of theoretical and experimental results for Suprasil W in the incoherent regime $T>T^{*}$. The experimental values are taken from [14]

\begin{tabular}{|c||c|c|}
\hline & Experiment & Theory \\
\hline$Q^{-1}$ & $(7 \pm 2) \times 10^{-5} T / \mathrm{K}$ & $6.1 \times 10^{-5} \mathrm{~T} / \mathrm{K}$ \\
\hline$\delta v / v$ & $2 \times 10^{-5} T / \mathrm{K} \ln \left(1.6 \times 10^{-13} \omega / 2 \pi \mathrm{Hz}\right)$ & $2.2 \times 10^{-5} T / \mathrm{K} \ln \left(T^{*} \hbar \omega /\left(k_{B} T^{2}\right)\right)$ \\
\hline
\end{tabular}

